\title{
The Perirhinal Cortex Modulates V2 Activity in Response to the Agreement Between Part Familiarity and Configuration Familiarity
}

\author{
Mary A. Peterson, ${ }^{1,2 *}$ Laura Cacciamani, ${ }^{1}$ Morgan D. Barense, ${ }^{3,4}$ and Paige E. Scalf ${ }^{1,2}$
}

\begin{abstract}
Research has demonstrated that the perirhinal cortex (PRC) represents complex object-level feature configurations, and participates in familiarity versus novelty discrimination. Barense et al. [(in press) Cerebral Cortex, 22:11, doi:10.1093/cercor/bhr347] postulated that, in addition, the PRC modulates part familiarity responses in lower-level visual areas. We used fMRI to measure activation in the PRC and V2 in response to silhouettes presented peripherally while participants maintained central fixation and performed an object recognition task. There were three types of silhouettes: Familiar Configurations portrayed realworld objects; Part-Rearranged Novel Configurations created by spatially rearranging the parts of the familiar configurations; and Control Novel Configurations in which both the configuration and the ensemble of parts comprising it were novel. For right visual field (RVF) presentation, BOLD responses revealed a significant linear trend in bilateral BA 35 of the PRC (highest activation for Familiar Configurations, lowest for Part-Rearranged Novel Configurations, with Control Novel Configurations in between). For left visual field (LVF) presentation, a significant linear trend was found in a different area (bilateral BA 38, temporal pole) in the opposite direction (Part-Rearranged Novel Configurations highest, Familiar Configurations lowest). These data confirm that the PRC is sensitive to the agreement in familiarity between the configuration level and the part level. As predicted, V2 activation mimicked that of the PRC: for RVF presentation, activity in V2 was significantly higher in the left hemisphere for Familiar Configurations than for Part-Rearranged Novel Configurations, and for LVF presentation, the opposite effect was found in right hemisphere V2. We attribute these patterns in V2 to feedback from the PRC because receptive fields in V2 encompass parts but not configurations. These results reveal two new aspects of PRC function: (1) it is sensitive to the congruency between the familiarity of object configurations and the parts comprising those configurations and (2) it likely modulates familiarity responses in visual area V2. ๑ 2012 Wiley Periodicals, Inc.
\end{abstract}

KEY WORDS: medial temporal lobe; visual perception; feedback; figure-ground; laterality

\section{INTRODUCTION}

Traditionally, the perirhinal cortex (Brodmann's cytoarchitectonic areas 35 and 36) of the medial temporal lobe is believed to be necessary for

\footnotetext{
${ }^{1}$ Department of Psychology, University of Arizona, Tucson, Arizona; ${ }^{2}$ Cognitive Science Program, University of Arizona, Tucson, Arizona; ${ }^{3}$ Department of Psychology, University of Toronto, Toronto; ${ }^{4}$ Rotman Institute, Toronto

Grant sponsor: NSF; Grant number: BCS 0960529 (to M.A.P.); Grant sponsor: CIHR; Grant number: MOP-115148 (to M.D.B.); Grant sponsor: McKnight Brain Research Foundation Grant sponsor: NSERC Discovery Grant (to M.D.B.)

*Correspondence to: Mary A. Peterson, Department of Psychology, 1503 E. University Blvd., University of Arizona, Tucson, AZ 85721, USA.

E-mail: mapeters@email.arizona.edu

Accepted for publication 27 July 2012

DOI 10.1002/hipo.22065

Published online in Wiley Online Library (wileyonlinelibrary.com).
}

declarative memory, but unnecessary for perception (e.g., Squire and Zola-Morgan, 1991; Suzuki, 2009; Clark et al., 2011; Kim et al., 2011; Squire and Wixted, 2011). In recent years, however, an alternative view has emerged. This view holds that the perirhinal cortex (PRC) represents complex object-level configurations assembled from features represented at lower levels of the visual hierarchy, and that these complex configurations subserve both perception and memory (e.g., Murray et al., 2007; Baxter, 2009; Cowell et al., 2010a; Graham et al., 2010; Lee et al., 2012; Murray and Wise, 2012). Accumulating evidence supports this alternative view; however, many of the tasks used to test perception have a substantial working memory component and thus, may support the less transformational view that the PRCs role extends to working memory but not to perception.

Recently, Barense et al. (in press) showed that the PRC plays a role in a quintessentially perceptual function: figure-ground perception. Figure-ground perception entails differentiating visual scenes into separate objects, and in particular, assigning a border shared by two regions to only one of them; that region is the "figure" whereas the abutting region appears to be simply the figure's local background. Barense et al. assessed figure-ground perception using a task that measures effects of "configuration familiarity" on figure assignment (Peterson et al., 1998, 2000; Gibson and Peterson, 1994; Peterson and Gibson, 1994a,b). In this task, participants report whether they perceive the figure on the left or the right side of a central border (see Fig. 1). There are two types of displays. In one type, a critical region on one side of the border portrays a Familiar Configuration (i.e., a portion of a well-known real-world object, Figs. 1A-C) in its typical upright orientation. In the other type, the critical region is a matched, equal-area region that portrays a novel object created by spatially rearranging the (familiar) parts of the upright familiar configuration (Part-Rearranged Novel Configurations; Figs. 1D-F). Critical regions in the Familiar Configurations condition are typically perceived as figures substantially and significantly more often than critical regions in the Part-Rearranged Novel Configurations condition, even though both of these conditions are comprised of the same ensemble of parts. Consequently, Peterson et al. (1991) concluded that configuration familiarity, but not part familiarity, serves as a cue to figural status. Because configuration familiarity operates implicitly in 

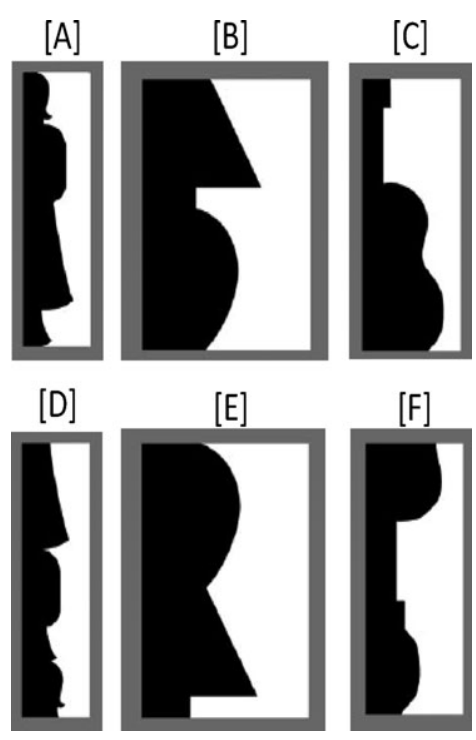

FIGURE 1. Stimuli Barense et al. (in press) used to test effects of configuration familiarity on figure assignment. The critical regions are shown in black on the left. (A-C) The critical regions depict the intact configuration of familiar, real-world, objects (from left to right: a woman, a lamp, and a guitar). (D-F) The critical regions are formed by spatially rearranging the parts of the familiar configurations in (A-C), respectively, such that the configuration is novel but the parts are the same. Participants' task was to indicate whether they saw the black or white region as figure. Black/white color and left/right location of critical regions were balanced in the experiment. Reprinted with permission from Barense et al., Cereb Cortex, (in press) New York, NY: Oxford University Press.

figure-ground perception (Peterson et al., 2000; Peterson and Skow, 2008) and because the effects occur at the configuration level (rather than the part level), the figure-ground task is an ideal probe of whether the PRC plays a role in perception (Barense et al., in press).

Barense et al. (in press) tested two patients with medial temporal lobe (MTL) damage that included the PRC; these patients failed to show an effect of configuration familiarity on figure assignment. Both age-matched control participants and two patients with damage limited to the hippocampus only (and not other MTL areas) showed typical effects of configuration familiarity on figure assignment. Interestingly, the performance of MTL-damaged patients deviated from control performance in two ways: first, they reported seeing the Familiar Configurations as figure somewhat less often than controls, and second, they reported seeing the Part-Rearranged Novel Configurations as figure more often than controls. Taken alone, the first finding is consistent with the theoretical view that the PRC of the MTL contains representations of complex configurations (Bussey et al., 2002; Lee et al., 2005; Barense et al., 2005, 2007, 2010a; Bartko et al., 2007; Lee and Rudebeck, 2010; Burke et al., 2011), and damage to these configural representations removes effects of configuration familiarity on figure assignment. Taken together, however, the two findings suggest that either output from the PRC is privileged over that from lower-level visual regions during figure-ground assignment (a feedforward explanation for the data) or that the intact PRC plays a role in modulating processing in lower-level visual areas (a feedback explanation).

On the feedforward view, non-brain-damaged individuals do not show effects of familiar parts when they are arranged in a novel configuration because the "novelty" response to the configuration in the PRC would outweigh the "familiarity" response to the parts in lower-level visual cortex. If the PRC were damaged (and thus, silenced) the weights of the "familiarity" responses in lower-level visual cortex would be increased, allowing them to influence figure-ground assignment. This feedforward view is consistent with computational models of PRC function (e.g., Bussey and Saksida, 2002; Cowell et al., 2006, 2010b; for review, see Cowell, 2012); to date, these models do not posit feedback to lower-level visual areas.

On the feedback view, non-brain-damaged individuals do not show effects of familiar parts when those parts are arranged in a novel configuration because the intact PRC sends differential feedback to lower-level visual areas sensitive to part familiarity when it detects a novel configuration composed of familiar parts versus a familiar configuration. Specifically, Barense et al. (in press) proposed that when the PRC detects a configuration composed of familiar parts in a novel spatial arrangement, it suppresses activation in lower-level visual areas sensitive to part familiarity, whereas when it detects a familiar configuration (by definition composed of familiar parts), it facilitates activation of lower-level visual areas sensitive to part familiarity (see Fig. 2). As a result of this interaction familiarity/novelty responses in lower-level visual regions and higherlevel MTL regions are coordinated to drive a coherent behavioral response. This feedback interpretation builds on evidence that the PRC discriminates between novel and familiar objects (Xiang and Brown, 1998; Henson et al., 2003; Kohler et al., 2005; Albasser et al., 2010; Burke et al., 2010; McTighe et al., 2010). The feedback framework explains why effects of part familiarity cannot be observed independently of effects of configuration familiarity in behavioral tasks conducted with nonbrain-damaged participants: when the PRC is intact, part familiarity responses at lower-levels are suppressed when familiar parts appear in Part-Rearranged Novel Configurations. On this view, effects of familiar parts were evident in tests of PRC-damaged participants because their PRC damage removed the suppression of part familiarity responses at lower levels in the visual hierarchy.

Discriminating between the feedforward and feedback models is important not only for understanding the role of the PRC in perception but also for current debates regarding whether visual perception requires only feedforward processes (Kirchner and Thorpe, 2006; Serre et al., 2007) or requires both feedforward and feedback processes (e.g., Lamme and Roelfsema, 2000; Bullier, 2001; Peterson and Skow, 2008). In the present experiment, we investigated whether responses to familiar parts in a lower-level visual area are modulated as a function of their configural arrangement (arranged in a Familiar Configuration or in a Part-Rearranged Novel Configuration), as predicted by Barense et al. (in press). Such a finding 
[A] Familiar Configuration

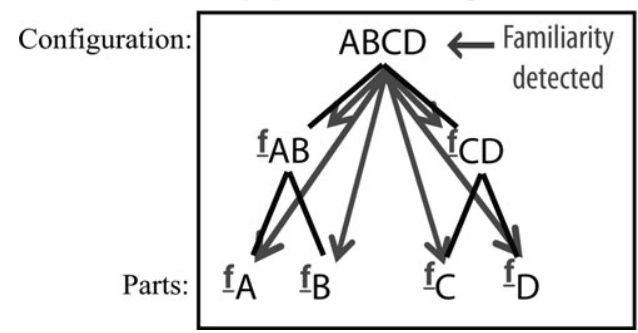

[B] Part-Rearranged Novel Configuration

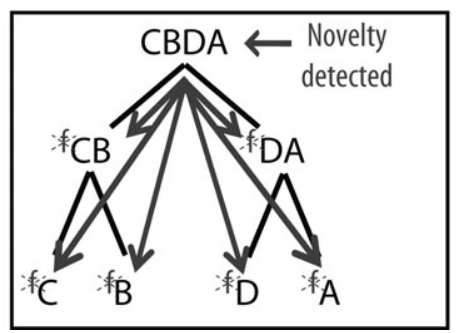

FIGURE 2. Schematic illustration of Barense et al.'s (in press) proposed role of the PRC in coordinating familiarity/novelty responses at high and low levels. (A) The PRC detects a familiar configuration and sends feedback (shown by arrows) to low levels, facilitating the part familiarity response (shown by underlined

in a region of visual cortex in which receptive fields are small and can encompass only object parts could not occur in the absence of feedback from a region sensitive to configural familiarity. We investigate the possible locus of such a region, which, as mentioned previously, is likely to both be sensitive to the configural arrangement of familiar parts and to lie within the cortical regions of the MTL.

Participants made object recognition judgments regarding the Familiar Configurations and the Part-Rearranged Novel Configurations used by Barense et al. (in press) while undergoing functional magnetic resonance imaging (fMRI). For the present experiment, we altered the displays to ensure that the critical region (i.e., the Familiar Configuration or the PartRearranged Novel Configuration) was perceived as figure: the critical regions were both higher in contrast against the overall screen background and much smaller in area than abutting regions (see also Driver and Baylis, 1996; Peterson and Enns, 2005; Barense et al., in press, Experiment 3). Samples are shown in the first and third rows of Figure 3. Participants fixated a central cross while viewing the displays; hence, the critical regions were lateralized in either the left or the right visual field.

We examined blood-oxygen-level-dependent (BOLD) signal in both PRC and V2, a visual area whose small receptive fields (approximately $2^{\circ}$ at $4^{\circ}$ eccentricity in the upper visual field; Kastner et al., 2001; Bles et al., 2006; Scalf and Beck, 2011) are likely to encompass the constituent parts, but not the configural wholes, of our lateralized stimuli. Employing lateralized visual stimuli allowed us to accurately locate their representations within V2. Furthermore, stimuli projected to different visual fields can be analyzed separately, allowing us to test for any possible hemispheric difference in configural processing. We anticipated that the PRC would show greater activation to Familiar Configurations than to Part-Rearranged Novel Configurations. We also anticipated that V2 would show greater activation to familiar parts in Familiar Configurations than to the same familiar parts in Part-Rearranged Novel Configurations. As mentioned previously, due to small receptive field sizes, such a pattern could not occur in V2 in the absence of feedback; a region in PRC showing a similar pattern would be a candidate bold fs). (B) The PRC detects a novel configuration and inhibits part familiarity responses at low levels (shown by crossed-out fs). Reprinted with permission from Barense et al., Cereb Cortex, (in press) (C) New York, NY: Oxford University Press.

source of such feedback. Furthermore, such a region in PRC should be sensitive to the familiarity versus novelty of both configurations and the ensemble of parts comprising them. To ensure that this was the case for any PRC region identified in our analysis, we included a control condition created by presenting the Part-Rearranged Novel Configurations in an inverted orientation. For these figures, neither the configurations nor the ensemble of parts was familiar. In the remainder of this paper, we refer to these displays as Control Novel Configurations (samples are shown in the middle row of Fig. 3).

Although others have explored interactions between the PRC and high levels in the visual processing hierarchy (area TE) (Higuchi and Miyashita, 1996; Liu and Richmond, 2000; Naya et al., 2001, 2003; Takeuchi et al., 2011), to our knowledge, this experiment is the first to examine whether PRC responses covary with those in low-level visual areas. It is also the first experiment that directly investigates the existence of perceptual asymmetries in processing configural familiarity.

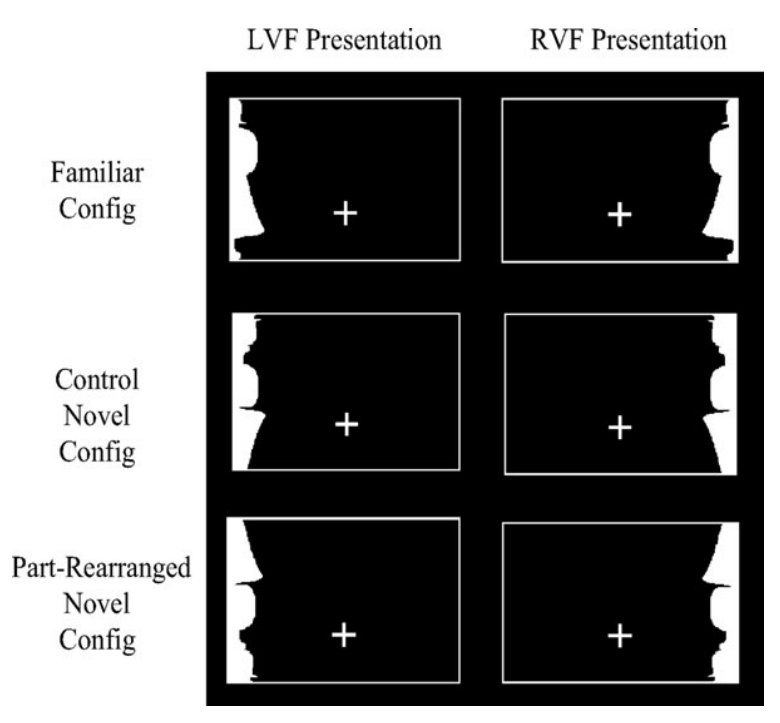

FIGURE 3. The six experimental conditions. The exemplar shown here is the standing woman. LVF = left visual field. RVF = right visual field. Config $=$ configuration. 


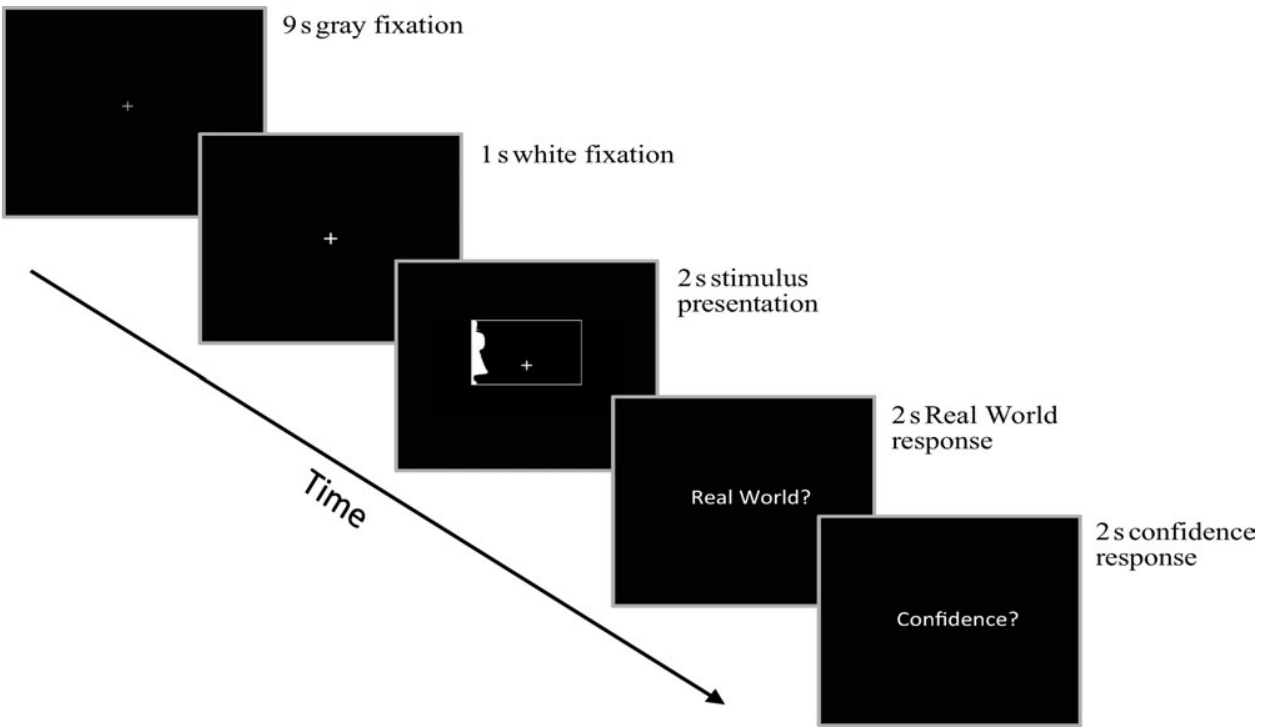

FIGURE 4. Trial structure. A sample trial in the LVF Familiar Configuration condition is shown here.

\section{MATERIALS AND METHODS}

\section{Participants}

We tested eight volunteers (5 males; ages 19-38), all with normal or corrected-to-normal visual acuity. Participants gave written informed consent to participate in this study, which was approved by the Institutional Review Board of the University of Arizona. Two participants were eliminated due to high error ( $>33 \%$ incorrect, which is greater than two standard deviations from the mean error score of the usable participants); thus, data from six participants (three males) were subjected to the analysis described below.

\section{Stimuli}

The stimuli were contrast-reversed versions of those by Barense et al. (in press) used in Experiment 3 (see Fig. 3). All stimuli were white silhouettes presented on a black screen with a thin white rectangular border. A white fixation cross was displayed in the center of the screen during stimulus presentation, and the white silhouette and its border were shifted up $1.35^{\circ}$ from this central fixation in order to better target the ventral visual stream. Silhouettes subtended $6.5^{\circ}$ in height and an average of $2.95^{\circ}$ in width, and the center-most edge of each figure was always located $3.5^{\circ}$ from fixation. These elongated, lateralized displays both caused the critical region to be assigned figural status and allowed retinotopic assignment of striate and extrastriate visual representations.

We used stimuli of three configuration types (24 unique stimuli per configuration type): Familiar Configurations, PartRearranged Novel Configurations, and Control Novel Configurations (see Fig. 3). The Familiar Configuration silhouettes portrayed portions of objects whose configurations and parts were likely to be known to participants (i.e., a standing woman, lamp, guitar; see Peterson et al., 2000). The Part-Rearranged Novel Configuration silhouettes were created by dividing the Familiar Configurations into parts at minima of curvature and spatially rearranging the parts to form a novel configuration; for these stimuli, the ensemble of parts had been seen together frequently, but in a different configuration. The Control Novel Configuration silhouettes were created by inverting the PartRearranged Novel Configuration silhouettes; consequently both the ensemble of parts and their configuration were novel. Each stimulus was presented twice; once in the right visual field (RVF) and once in the left visual field (LVF). There were therefore six stimulus categories in total, with 24 stimuli in each category: LVF Familiar Configurations, RVF Familiar Configurations, LVF Part-Rearranged Novel Configurations, RVF Part-Rearranged Novel Configurations, LVF Control Novel Configurations, and RVF Control Novel Configurations.

\section{Experimental Design and Equipment}

Because our number of stimuli was small, we employed a slow event-related trial design (see Fig. 4). Each trial began with a 10-s fixation period; one second before stimulus onset, the fixation cross brightened from gray to white to alert the participant to the upcoming stimulus. Participants were instructed to maintain fixation on this white cross during the 2-s presentation of the stimulus item. The query "Real World?" then appeared for $2 s$ at fixation, prompting the participant to indicate whether the silhouette depicted a real-world or novel object using a button-box held in the their right hand, with one button assigned to "yes/real-world object" and the other assigned to "no/novel object." The query "Confidence?" then appeared at fixation for $2 \mathrm{~s}$, prompting participants to report their confidence in their "real world" judgment using a buttonbox held in their left hand, with one button assigned to "high confidence" and the other assigned to "low confidence." 
Participants' responses were collected using two Lumina (Cedrus Corp) response pads and controller. Participants viewed the stimuli through goggles designed by Magnetic Resonance Technologies. Vision Egg (Straw, 2008) software running under Windows XP Pro with SP3 on a Dell Optiplex 380 Pentium Dual-Core CPU $5400 @ 2.70 \mathrm{GHz}$ controlled stimulus presentation and response collection.

Participants performed six runs of 24 trials each. In each run, half of the images appeared in the RVF and half appeared in the LVF. One-third of the images in each run were Familiar Configurations, one-third were Part-Rearranged Novel Configurations and one-third were Control Novel Configurations. A Latin square design was used to assign individual images to each run; one and only one version of the six possible stimulus categories (i.e., LVF Familiar Configurations, RVF Familiar Configurations, LVF Part-Rearranged Novel Configurations, RVF Part-Rearranged Novel Configurations, LVF Control Novel Configurations, RVF Control Novel Configurations) of each stimulus exemplar appeared in each of the six runs (e.g., the standing woman appeared in only one of those six versions in a given run). Due to the slow event-related design used in this experiment, all stimuli were randomized within each run.

\section{Practice and Eye-Fixation Training}

Within the $24 \mathrm{~h}$ before the scanning session, each volunteer participated in a training session outside of the scanner. During this session, participants viewed multiple examples of "realworld" and "novel" stimuli, and were instructed to categorize the subsequent stimulus items on this basis. Participants were told that real-world silhouettes clearly portrayed namable, every-day objects and that novel silhouettes, in contrast, were created in the lab, and would have only poor (if any) resemblance to any every-day namable objects.

Participants were given 24 practice trials (stimuli were different from those used during fMRI scanning) and received feedback on their performance. Critically, eye movements were monitored during these practice trials using an Eyelink 2000 eye-tracker on a Pentium 4 Dell PC running Windows XP Professional; if a participant deviated from fixation during the 2-s presentation of a silhouette, an error tone was played, and the trial was aborted and re-presented. This quickly trained participants to fixate on the cross and resist the urge to look toward the stimulus appearing in the periphery. Training was completed only when the participant had performed all 24 trials while maintaining fixation. Participants were reminded of this eye-fixation training session immediately before the experimental scanning session, but eye movements were not monitored in the scanner.

\section{Data Acquisition and Analysis}

Images were collected on a 3.0 T Signa whole-body scanner (Signa Echo Speed; General Electric, Milwaukee, WI) using a four-channel phased array head coil. We used an EPI spiral pulse sequence $(\mathrm{TR}=2,000, \mathrm{TE}=30, \mathrm{FOV}=240 \times 240$ $\mathrm{mm}, 5 \mathrm{~mm}$ with a $0.6 \mathrm{~mm}$ skip) to acquire 30 oblique slices (rotated $13^{\circ}$ from the anterior commissure-posterior commissure plane). Each of the six scanning runs contained 197 repetitions. To help register functional scans to anatomical space, we also acquired a T2-FLAIR (fluid attenuated inversion recovery) anatomical image in plane with the functional scans. Additionally, we collected four high-resolution T1-weighted SPGR images during retinotopic scanning (see below) which were used for coregistration.

We used FMRIB (Oxford University Centre for Functional MRI of the Brain) Software Library (FSL) to analyze our functional data. Data were brain-extracted, high-pass filtered (sigma $=50 \mathrm{~s}$ ), intensity-normalized, and motion-corrected [FSL 4.1.9, (Jenkinson et al., 2002; Smith et al., 2004)].

We concatenated data across all six runs, and submitted them to GLM analysis using FEAT (FMRI Expert Analysis Tool) v 5.98 [FSL 4.1.9; (Woolrich et al., 2001; Smith et al., 2004)]. We modeled six regressors of interest corresponding to our six categories of stimuli (RVF Familiar Configurations, RVF Part-Rearranged Novel Configurations, RVF Control Novel Configurations, LVF Familiar Configurations, LVF PartRearranged Novel Configurations, LVF Control Novel Configurations). These regressors were convolved with a doublegamma model of the HRF (Phase 0s). In order to equate the number of trials in each condition, we included in a regressor of no interest not only items that were incorrectly categorized in one of its permutations (e.g., an RVF Familiar Configuration item called "novel"), but also their counterparts in their other permutations (e.g. the corresponding RVF Part-Rearranged Novel Configuration and RVF Control Novel Configuration). We note that this regressor was designed to simultaneously equate statistical power across conditions of interest as well as to remove contaminating error trials from the error term of our GLM; the regressor in and of itself has no theoretical meaning. Condition specific error trials were of insufficient power (less than eight per cell) to merit individual modeling. The resulting statistical maps were registered into the participant's individual anatomical space and into standard space using FMRIBs Linear Image Registration Tool (FLIRT; Jenkinson et al., 2002).

\section{Medial Temporal Lobe Analysis Procedures}

The statistical maps for RVF and LVF stimulation were fed into separate higher-level ordinary least squares (OLS) group analysis by FMRIBs Local Analysis of Mixed Effects (FLAME). Because we were looking for the region of PRC specifically sensitive to the conjunctive status of configural familiarity and part familiarity (and thus able specifically to prevent Part-Rearranged Novel Configurations from being recognized as "familiar"), our regressor of interest was a linear model (Familiar Configuration, Control Novel Configuration, Part-Rearranged Novel Configuration; vector $-1,0,1)$. Specifically, we used the Control Novel Configuration as baseline against which to judge the relative activation of Familiar Configuration and Part-Rearranged Novel Configuration. This linear contrast assured us that difference in activation between the two 
conditions reflected differential response to the arrangement of familiar parts, rather than simply reflecting a novel configuration of any kind. We also modeled out each participant's mean activation level as a regressor of no interest. We modeled data from each visual field in separate analyses because their inclusion in a common analysis would have altered the "baseline" (meant to be novel configurations from the appropriate visual field) of the linear trend we intended to test.

We used Brodmann areas (BA20, BA28, BA35, BA36, and BA38) from the Talairach-to-MNI-conversion digital atlas (Lancaster et al., 2000) to identify regions of interest (ROIs) in the MTL that correspond with the areas that often accompany perirhinal damage in patients. Perirhinal cortex is typically defined as BA 35 and 36, although BA 38 has many of the same cytoarchitectonic characteristics as PRC (Suzuki and Amaral, 1994) and is sometimes included with PRC (see Suzuki and Amaral, 1994 and the "total perirhinal cortex" measure in Insausti et al., 1998). Because the Talairach-to-MNI-conversion digital atlas strictly limits Brodmann areas to regions that correspond to cortical bark in MNI 152 space, these areas are narrower than the spatial resolution we could achieve. In order to produce more usable masks for group analysis, we projected the Talairach conversion masks into each participant's functional space, transformed the resulting (larger) probabilistic map into standard MNI space, and then averaged these transformations across all participants. This produced larger ROIs that were both more appropriate for analyzing data of our spatial resolution and directly reflected the individual anatomical spaces of our subject population. We then used easythresh (FEAT 5.98) to identify clusters of voxels $(Z>1.96)$ showing a linear trend whose extent was greater than would be predicted by random variation in activation $(P<0.05)$.

\section{Visual Cortex Analysis Procedures}

Each volunteer also participated in a separate retinotopic mapping session. The procedures used during this session are the same as those described in Scalf and Beck (2010) and are derived from those established by Sereno et al. (1995) and adapted by Kastner et al. (1998). Briefly, each quadrant of visual cortex (upper/lower, left/right) represents $90^{\circ}$ of the visual field in a retinotopic manner. V1 through V4 may be identified by locating the regions that represent the boundaries of these areas (either $0^{\circ}$ or $90^{\circ}$ on the polar azimuth); once each region of visual space has been represented in one area, the retinotopic mapping "reverses" and the subsequent visual area represents visual space in a "mirror reversed" manner. We compared activation between stimulation of the horizontal and vertical meridians of the visual field to identify "reversals." We compared activation between upper and lower visual field stimulation to functionally segregate ventral and dorsal striate and extrastriate cortex. During this session, EPI spiral pulse sequences $(\mathrm{TR}=$ $2,000, \mathrm{TE}=30, \mathrm{FOV}=240 \times 240 \mathrm{~mm}$, slice thickness $=2$ $\mathrm{mm} ; 30$ oblique slices rotated $13^{\circ}$ from the anterior commissure-posterior commissure plane) were used to collect six runs of 105 repetitions and six runs of 95 repetitions. Four high-re- solution $(1 \mathrm{~mm})$ SPGR T1-weighted images were collected to submit to Freesurfer (Dale et al., 1999; Fischl et al., 1999, 2001) for averaging, segmentation, and flatmapping. Further details of retinotopic mapping and analysis are identical to those reported by Scalf and Beck (2010).

Because a group analysis would average together striate and extrastriate regions with different receptive field sizes, we employed an ROI analysis to interrogate visual cortex. We used activation to the Control Novel Configuration condition in the contralateral visual field to identify regions in right and left V1 through V4 stimulated by our bipartite displays. Striate and extrastriate activation to the Control Novel Configuration condition should also have been unmodulated by MTL structures (which were silent during this condition), so ROIs identified by this condition should have been relatively unbiased by potential feedback from the MTL. Our measure of interest in visual cortex was the degree to which each regressor (parameter) predicted change in the BOLD signal; these measures are usually referred to as "parameter estimates." We note that that percent signal change is often the measure of interest in such analyses. Although there are a number of ways to calculate percent signal change, nearly all of them involve scaling the parameter estimates by the inverse of the mean signal; some also use additional scaling factors. Rather than relying on a somewhat arbitrary method of scaling our parameter estimates, we chose to simply use the unscaled values as our measure of interest.

Parameter estimates from the Control Novel Configuration conditions for each visual field were projected onto the appropriate hemisphere in Freesurfer space. Suprathreshold $(Z>2)$ voxels that were contiguous with the peak activation in each region of visual cortex were assigned to that region's ROI. The masks for each participant were then projected back into their individual high-resolution anatomical space in FSL. We then used Featquery (Smith et al., 2004) to extract the mean parameter estimates for the Familiar Configuration and the Part-Rearranged Novel Configuration conditions from the ROIs in each region of visual cortex. During this process, Featquery applied the SPGR to EPI transformation matrix calculated during image registration to determine which EPI voxels fell within the ROI (selected in MPRAGE space).

\section{RESULTS}

\section{Behavioral Results}

We subjected the accuracy data to repeated measures analysis of variance (ANOVA) using the factors Visual Field (LVF, RVF) and Configuration Type (Familiar Configuration, Control Novel Configuration, Part-Rearranged Novel Configuration). A significant main effect of condition was found, $F_{(10,2)}$ $=24.79 ; P<0.001$, with participants less accurate in "real world" than "novel" judgments (see Table 1). This effect may be due to response bias; $66 \%$ of the trials were novel, thus biasing participants to respond "novel" more often than "real 
TABLE 1

Mean Accuracy, RT (correct trials only), and Confidence Scores for Each Condition (SEM in Parentheses)

\begin{tabular}{|c|c|c|c|c|c|c|}
\hline & \multicolumn{2}{|c|}{ Familiar Config } & \multicolumn{2}{|c|}{ Control Novel Config } & \multicolumn{2}{|c|}{ Part-Rearranged Novel Config } \\
\hline & LVF & RVF & LVF & RVF & LVF & RVF \\
\hline Proportion correct & $0.65(0.03)$ & $0.67(0.03)$ & $0.87(0.05)$ & $0.88(0.04)$ & $0.85(0.04)$ & $0.85(0.04)$ \\
\hline $\mathrm{RT}(\mathrm{ms})$ & $2,051(267)$ & $2,070(284)$ & $2,008(281)$ & $1,945(343)$ & $1,994(352)$ & $1,956(324)$ \\
\hline Confidence $(1=$ low, $2=$ high $)$ & $1.85(0.08)$ & $1.82(0.09)$ & $1.56(0.15)$ & $1.67(0.13)$ & $1.70(0.12)$ & $1.67(0.12)$ \\
\hline
\end{tabular}

world." Alternatively, explicit recognition may have been difficult, given that the stimuli were peripherally presented silhouettes, which portrayed only portions of real-world objects, and, as silhouettes, lacked surface details and features. Together, these factors may have led participants to set a high threshold for responding "real world."

A repeated measures ANOVA on the confidence ratings on accurate trials using Visual Field (RVF, LVF) and Configuration Type (Familiar Configuration, Part-Rearranged Novel Configuration, Control Novel Configuration) as factors revealed a significant main effect of condition, $F_{(5,1)}=9.15 ; P<0.01$ (see Table 1). Paired-samples $t$-tests further showed that when subjects responded "real world" accurately to Familiar Configurations, they were significantly more confident than when they responded "novel" to Control Novel Configurations, $t_{(5)}=$ 3.36; $P<0.05$, and marginally more confident than when they responded "novel" to Part-Rearranged Novel Configurations, $t_{(5)}=2.47 ; P=0.057$. Thus, participants were overall more confident in their "real world" response on trials with Familiar than Novel Configurations. Additionally, participants were marginally more confident when they responded "novel" accurately to Part-Rearranged Novel Configurations than to Control Novel Configurations, $t_{(5)}=2.22 ; P<0.08$. Confidence ratings, then, gave no evidence of any lingering realworld response to the ensemble of parts in the Part-Rearranged Novel Configurations condition.

We subjected RT data (from five participants, RT data from one participant were lost due to computer malfunction) from correct trials only to a repeated measures ANOVA using the factors Visual Field (LVF, RVF) and Configuration Type (Familiar Configuration, Control Novel Configuration, and Part-Rearranged Novel Configuration). No main effects or interactions approached conventional levels of significance $(P>0.1)$ (see Table 1$)$.

\section{Medial Temporal Lobe Analysis Results}

RVF stimulation produced significant clusters of voxels showing a linear response trend (Familiar Configuration > Control Novel Configuration $>$ Part-Rearranged Novel Configuration) in both left and right BA 35 (Num voxels $=270$, $\max Z=3.93, P<0.03$; Num voxels $=194, \max Z=3.36$, $P<0.05$, respectively), as shown in Figure 5A. Thus, when stimuli were presented in the RVF, bilateral BA 35 (PRC) showed the highest activation to Familiar Configurations, less activation to Control Novel Configurations, and the lowest activation to Part-Rearranged Novel Configurations (Fig. 5B). No such pattern emerged for stimuli presented to the LVF.

LVF stimulation did produce significant clusters of voxels showing a linear response trend in the opposite direction (PartRearranged Novel Configurations $>$ Control Novel Configurations $>$ Familiar Configurations) in both left and right BA 38 (Num voxels $=64$, $\max Z=2.76, P<0.04$; Num voxels $=$ 51, $\max Z=3.08, P<0.04$, respectively), as shown in Figure 5C. Thus, when stimuli were presented to the LVF, bilateral BA 38, which corresponds to the temporal pole of the MTL (adjacent to area 36 of the PRC), showed the most activation to Part-Rearranged Novel Configurations, less activation to Control Novel Configurations and the lowest activation to $\mathrm{Fa}-$ miliar Configurations, as shown in Figure 5D.

No other BAs in the MTL exhibited linear trends that approached conventional levels of significance.

\section{Visual Cortex Analysis Results}

We subjected parameter estimates from the right and left presentations of Familiar Configurations and Part-Rearranged Novel Configurations from the ROIs in each region of visual cortex in each hemisphere of each participant to a repeated measures ANOVA with the factors Visual Field (RVF, LVF) and Configuration Type (Part-Rearranged Novel Configuration, Familiar Configuration). (Recall that Control Novel Configuration parameter estimates from each visual field were not eligible for analysis because they were used to establish our ROIs in each region of visual cortex.) As can be seen in Table 2, participants showed greater activation in left hemisphere (LH) visual cortex when the stimuli fell within the right (contralateral) visual field, and in right hemisphere $(\mathrm{RH})$ visual cortex when the stimuli fell within the left (contralateral) visual field. Taken together, these effects indicate that participants were successful at maintaining fixation on the center cross during stimulus presentation. Had participants moved their eyes toward each stimulus rather than fixating on the central cross, each stimulus would have straddled the visual fields and been represented in both hemispheres, and consequently would have produced activation that was insensitive to its lateralized position in the display.

We found significant interactions between the factors Visual Field and Configuration Type in V2 in both the LH, $F_{(1,5)}=$ 17.00; $P=0.009$, and the RH, $F_{(1,5)}=7.22 ; P=0.043$, 


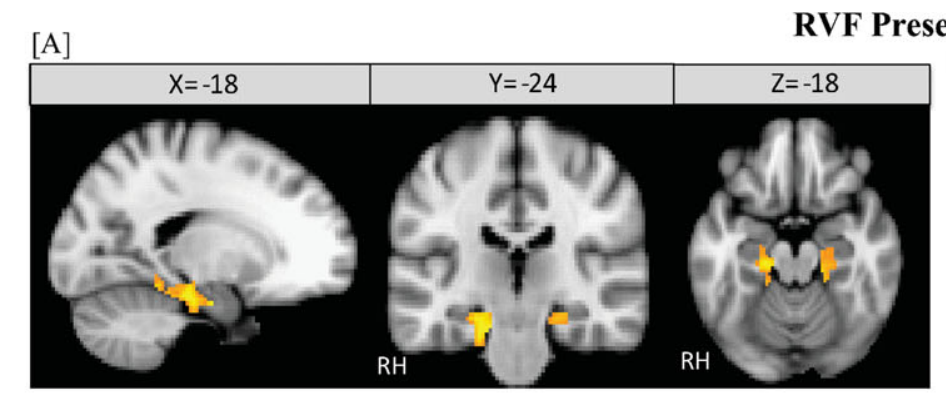

ntation: BA 35
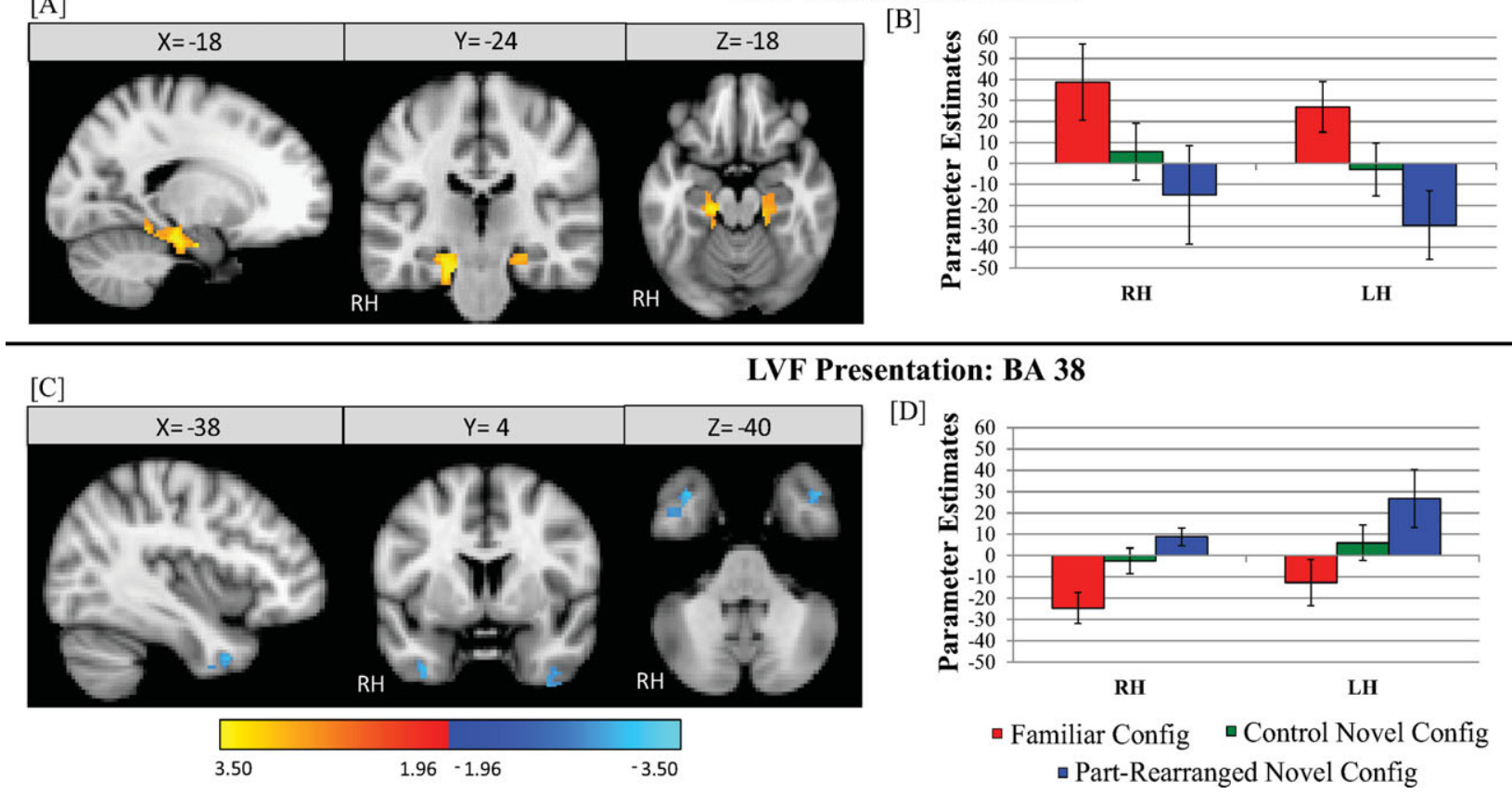

FIGURE 5. Effects of condition in the MTL. (A and B) BA 35 (PRC) activation for RVF presentation. (C and D) BA 38 (temporal pole) activation for LVF presentation. Statistical maps (A and C) were thresholded at $P<0.05$ and are shown superimposed on a standard brain in MNI space. The color bar reflects $z$-scores for the linear trend in the direction of Familiar Configurations $>$ Control Novel Configurations > Part-Rearranged Novel Configurations; thus, a negative score, as in (C), indicates a significant linear trend in the opposite direction. Parameter estimates (B and D) for each condition were extracted from these suprathreshold clusters in both the RH and LH. Error bars represent SEM. RH = right hemisphere. $\mathrm{LH}=$ left hemisphere. LVF = left visual field. RVF = right visual field.

shown in Figure 6. Planned comparisons indicated that, in $\mathrm{LH}$ V2, this interaction was driven by greater activation resulting from RVF Familiar Configurations than Part-Rearranged Novel Configurations, $P=0.027$, whereas in RH V2, this interaction was driven by greater activation resulting from LVF Part-Rear-

\section{TABLE 2.}

Mean Parameter Estimates in Visual Cortex for LVF and RVF Presentation Conditions

\begin{tabular}{llllrl}
\hline & & LVF & RVF & \multicolumn{1}{c}{$F$} & $P$ \\
\hline LH & V1 & 206 & $\mathbf{2 4 6}$ & 4.80 & 0.08 \\
& V2 & 162 & $\mathbf{2 2 3}$ & 9.44 & 0.03 \\
& VP & 165 & $\mathbf{2 2 8}$ & 31.17 & 0.00 \\
& V4 & 132 & $\mathbf{2 0 4}$ & 19.96 & 0.01 \\
RH & V1 & $\mathbf{2 4 6}$ & 179 & 7.95 & 0.04 \\
& V2 & $\mathbf{2 4 1}$ & 157 & 8.58 & 0.03 \\
& VP & $\mathbf{2 4 8}$ & 154 & 9.81 & 0.03 \\
& V4 & $\mathbf{2 3 5}$ & 159 & 7.20 & 0.04 \\
\hline
\end{tabular}

$F$ values and $P$ values for ANOVAs comparing the parameter estimates obtained with LVF and RVF presentation for each region in each hemisphere. Higher values are bolded. The direction of difference in each hemisphere shows that subjects were fixating. $\mathrm{LH}=$ left hemisphere. $\mathrm{RH}=$ right hemisphere. $\mathrm{LVF}=$ left visual field. RVF = right visual field. ranged Novel Configurations than Familiar Configurations, $P$ $=0.022$. Thus, in LH V2, activation mirrors activation seen

\section{V2}

$\square$ Familiar Config $\quad$ aPart-Rearranged Novel Config

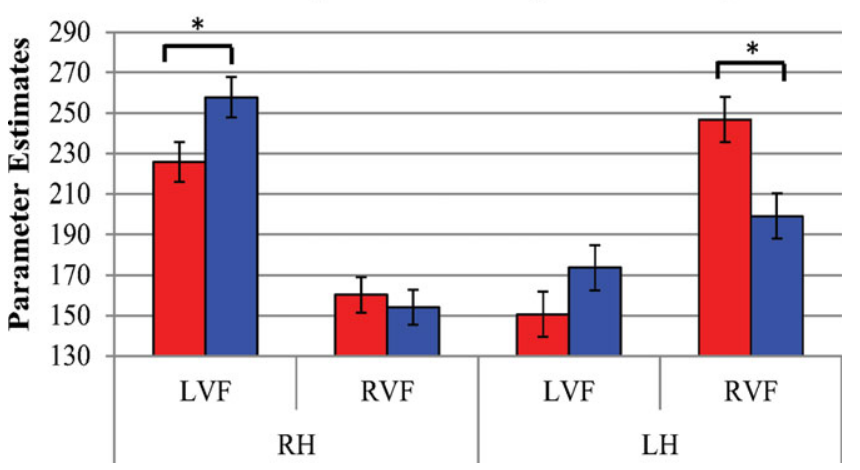

FIGURE 6. Effects of Visual Field and Configuration Type in V2. Parameter estimates were extracted from each subject's V2 ROI in each hemisphere, determined based on activation in the Control Novel Configuration condition for contralateral visual field presentation (thus, activation in this control condition was not eligible for visual cortex analyses). Error bars represent SEM of the difference between the conditions shown. $\mathrm{RH}=$ right hemisphere. $\mathrm{LH}$ $=$ left hemisphere. $\mathrm{LVF}=$ left visual field. $\mathrm{RVF}=$ right visual field. ${ }^{*} P<0.05$ 
in PRC BA 35 with RVF presentation, and in RH V2, activation mirrors activation seen in BA 38 with LVF presentation.

We also found a significant crossover interaction in LH V1, $F_{(1,5)}=12.42 ; P<0.02$. Planned comparisons showed that for LVF presentation, V1 was marginally less active to Familiar Configurations than Part-Rearranged Novel Configurations $(P=0.08)$, but for RVF presentation, V1 activation did not differ for Familiar Configurations versus Part-Rearranged Novel Configurations $(P=0.16)$. Because neither planned contrast was significant, this interaction was not given any further attention. No other region in visual cortex showed an interaction between Visual Field and Configuration Type that met or approached conventional levels of significance. The main effect of Configuration Type was not statistically significant.

An additional whole-brain analysis was also performed to assess whether a significant linear trend (in either direction) was evident in any other brain regions; no significant effects were found (cluster $P>0.05$; threshold $Z<2.3$ ).

\section{DISCUSSION}

We used fMRI to assess activation in the MTL and V2 while participants made object recognition judgments regarding three types of displays: Familiar Configurations (composed of familiar parts), Part-Rearranged Novel Configurations (novel configurations created by spatially rearranging the same familiar parts), and Control Novel Configurations (novel configurations composed of novel parts). Others have shown that the PRC responds more strongly to familiar than to novel configurations (e.g., Barense et al., 2011); we expected to replicate those findings. Furthermore, our previous findings (Barense et al., in press) led us to suspect that the PRC might be particularly important in adjudicating configural familiarity when the configuration's constituent parts are familiar rather than novel. We therefore included two novel configuration conditions; one in which the ensemble parts of the configuration were familiar and one in which they were novel (Part-Rearranged and Control Novel Configurations, respectively). Each type of configuration was presented in each visual field; this manipulation was necessary to identify the signal evoked by the items in striate and extrastriate cortex; it also allowed us to test for hemispheric differences in configural processing. We found that the hemisphere that initially processed the visual stimulus determined the region of anterior temporal cortex recruited to support task performance (i.e., BA 35 or 38), and determined the directionality of the response to configural familiarity. Both BA 35 and BA 38, however, responded differently as a function of the joint familiarity of the configuration and the ensemble of parts comprising the configuration. This is a new finding, uncovered because we manipulated the familiarity versus novelty of the ensemble of parts comprising our novel configurations. To our knowledge, no previous research has directly investigated this question.

\section{Medial Temporal Lobe Activation}

\section{RVF presentations}

For RVF presentations, BA 35, which corresponds to the PRC of the MTL, responded bilaterally. Relative to the control condition (novel configurations composed of a novel ensemble of parts), activation was increased when an ensemble of familiar parts was presented in its typical spatial arrangement (Familiar Configuration) and was decreased when an ensemble of familiar parts was presented in a novel spatial arrangement (PartRearranged Novel Configuration; Fig. 5). These results indicate that the PRC detects the familiarity of the ensemble of parts comprising a configuration as well as the familiarity of the configuration (i.e., the spatial arrangement of the parts) and responds to their congruency: responses are facilitated when both the parts and the spatial configuration of parts match in familiarity and are suppressed when the parts and spatial configuration of parts mismatch in familiarity (i.e., the ensemble of parts is familiar but the spatial arrangement is novel). As proposed by Barense et al. (in press), our results indicate that the PRC plays a critical role in discriminating familiar configurations from novel configurations composed by spatially rearranging an ensemble of familiar parts.

\section{LVF presentations}

For LVF presentations, BA 38, which corresponds to the temporal pole of the MTL (adjacent to BA 36 of the PRC), responded bilaterally. BA 38 also responded differentially to the Familiar Configurations and the Part-Rearranged Novel Configurations, but the pattern of activity was the opposite of that in BA 35. Relative to the Control Novel Configurations (novel configurations composed of a novel ensemble of parts), activation was increased when an ensemble of familiar parts was presented in a novel spatial arrangement and decreased when an ensemble of familiar parts was presented in its familiar spatial arrangement. Thus, BA 38 responded maximally to a mismatch in the familiarity of the ensemble of parts and the configuration.

\section{Visual Cortex Activation}

Responses in area V2 of the LH paralleled those observed in BA 35 with RVF presentation conditions: Responses were larger for Familiar Configurations than for Part-Rearranged Novel Configurations. Responses in area V2 of the RH, in contrast, paralleled those observed in BA 38 for LVF presentations: Here, responses were larger for Part-Rearranged Novel Configurations than for Familiar Configurations (Fig. 6). Given the size and eccentricity of our displays (Kastner et al., 2001; Bles et al., 2006; Scalf and Beck, 2011), V2 receptive fields encompass parts of our displays, but not configural wholes. Consequently, finding differential V2 responses to ensembles of familiar parts as a function of their configural familiarity is highly unlikely in the absence of modulation from other cortical regions. Furthermore, configural familiarity drives responses in 
LH and RH V2 in opposite directions. This pattern of results is extremely unlikely in the absence of feedback from higherlevel regions. We suspect that in the case of the LH, this function may be subserved by the PRC. Increased PRC activation to Familiar Configurations may result in enhanced LH V2 responses to the familiar parts, whereas decreased PRC activation to Part-Rearranged Novel Configurations may result in reduced LH V2 activation to familiar parts. Thus, BOLD responses to stimuli in the RVF are consistent with Barense et al.'s (in press) proposal that part-familiarity responses in lower-level brain regions are suppressed when an ensemble of familiar parts occurs in a novel configuration. RH V2, in contrast, may be modulated by BA 38's increased activity for familiar parts in novel configurations (i.e., Part-Rearranged Novel Configurations). In any case, the finding that the patterns of V2 response evoked by lateralized stimuli paralleled the pattern observed in the area of the MTL maximally activated under those presentation conditions is consistent with the feedback predictions made by Barense et al. (in press). This provides compelling evidence that MTL structures are employed in the service of perceptual tasks as well as memory tasks. These findings are also consistent with interactive models of perceptual function (Lamme and Roelfsema, 2000; Bullier, 2001; Peterson and Skow, 2008), and call for a modification to feedfowardonly models (e.g., Bussey and Saksida, 2002; Cowell et al., 2006; Serre et al., 2007).

The observation that top-down feedback from PRC suppressed part-based responses in lower-level visual regions is consistent with a recent series of findings suggesting that the PRC may be critical in resolving perceptual interference (McTighe et al., 2010; Burke et al., 2010; Romberg et al., 2012; Barense et al., 2012; Newsome et al., 2012). For example, patients with PRC damage were impaired at perceptual discrimination under conditions of high visual interference (a series of complex object discriminations for which object parts were repeatedly presented across trials), but performed normally under conditions of low visual interference (fewer object parts repeated across trials) (Barense et al., 2012; Newsome et al., 2012). These findings suggest that PRC damage rendered these individuals vulnerable to interference from object parts processed by lower-levels in the visual stream. The current results shed light on the mechanism involved: PRC damage removes topdown feedback that suppresses part-familiarity responses when parts appear in a novel configuration, thus ensuring that they do not interfere with performance.

\section{Using Lateralized Presentations}

The use of lateralized presentation methods with our particular stimuli in which we varied the familiarity of configurations and of the parts comprising them uncovered different responses in different regions of the anterior temporal lobes. Although the temporal pole (BA 38) is not usually considered to be PRC proper (but see Suzuki and Amaral, 1994 and the "total perirhinal measure" in Insausti et al., 1998), and our activation cluster was more lateral than what is typically considered PRC, it is usually not spared if the PRC is compromised. Consequently, it is worth investigating whether it plays a role in the perceptual processing deficits shown by patients with MTL damage. We therefore included it as an ROI in our analysis, and found that it was indeed sensitive to the familiarity of configurations of familiar part ensembles. Activation in the temporal pole is often associated with the processing of semantically or emotionally meaningful stimuli such as faces (e.g., Barense et al., 2011). Other evidence indicates a role for the temporal pole in accessing semantic information across a wide range of stimulus types (Bright et al., 2005; Lambon Ralph et al., 2008). Consistent with this, semantic dementia, a neurodegenerative condition characterized by progressive deterioration of semantic knowledge is associated with atrophy to the temporal poles (Bozeat et al, 2000; Rogers et al., 2004). We note that patients with semantic dementia are typically impaired at object decision (e.g., judging whether drawings represented real or nonsense objects; Patterson et al., 2006), as well as perceptual discrimination of complex objects (Barense et al., 2010b). As such, it seems likely they would have been impaired at the realworld decision task required of our participants. One intriguing possibility is that semantic processes were heavily recruited in resolving the ambiguous relationship among familiar parts that formed an unfamiliar whole. Regardless of the cause of temporal pole recruitment, however, its activation pattern covaried with that of $\mathrm{RH} V 2$, suggesting that semantic information about stimuli may modulate the signal they evoke at the earliest levels of visual processing.

Our findings also highlight a fact well known to clinical neuropsychologists but often forgotten by cognitive neuroscientists; the brain can employ any number of strategies to perform any given task. Lateralized stimulus presentation often encourages the brain to choose one particular strategy over another. Although both hemispheres are capable of performing nearly all tasks (auditory rhyming is a notable exception), they may use vastly different strategies to perform any single task. For example, stimuli presented to the LVF (RH) are more likely to be identified based on their concrete visual forms, while stimuli presented to the RVF (LH) are more likely to be identified on the basis of their abstract identity (Marsolek, 1999). Participants performing a rhythm-matching task appear to base their judgment on the absolute durations of the stimuli presented to the left ear (RH) and on the relative duration of the stimuli presented to the right ear (LH) (Scalf et al., 2009).

In our experiment, we have uncovered a difference in the neural substrates recruited to support judging the familiarity of a silhouette: stimuli presented to the RVF appear to be processed by the PRC while stimuli presented to the LVF appear to be processed by the temporal pole. Furthermore, familiarity is signaled differently by these two regions: for stimuli in the RVF, BA 35 increases signal for familiar conjunctions of familiar parts (Familiar Configurations), whereas for stimuli in the LVF, BA 38 increases signal for novel configurations composed of familiar parts (Part-Rearranged Novel Configurations). Clearly, the brain has dual routes to accessing information about previously encountered stimuli and their constituent 
parts. It behooves researchers to remember this when investigating "human patients with PRC damage," in whom compromised tissue often extends beyond the PRC.

Which hemisphere is in control of adjudicating configural familiarity in the neurologically intact brain? The problem of "metacontrol" rarely has a simple solution (Levy et al., 1976). In some cases, performance is dominated by whichever hemisphere is superior at the given task (e.g., Hellige et al., 1989). In other cases, task performance during bilateral trials looks nothing like task performance by either hemisphere in isolation (Banich and Karol, 1992). Finally, stimulus order and task conditions may influence which hemisphere dominates performance (Scalf et al., 2007). In the present study, we replicated previous evidence (Barense, et al., 2011) that PRC (BA 35) activation is higher for familiar than novel objects when we used RVF presentations, but we found the opposite pattern in the temporal pole (BA 38) when we used LVF presentations. It remains for future research to investigate the conditions that determine whether BA 35, BA 38, both regions, or some other region altogether, is recruited when subjects are asked to make real-world decisions regarding a centrally presented stimulus.

\section{Acknowledgments}

The authors thank Carol Barnes for her support and encouragement, Lee Ryan for her advice on design, and Lynn Nadel and the members of the University of Arizona Visual Perception and Cognition Lab Group and the Cognition and Neural Systems Seminar for their input.

\section{REFERENCES}

Albasser MM, Poirier GL, Aggleton JP. 2010. Qualitatively different modes of perirhinal-hippocampal engagement when rats explore novel vs. familiar objects as revealed by c-Fos imaging. Eur J Neurosci 31:134-147.

Banich MT, Karol DL. 1992. The sum of the parts does not equal the whole: Evidence from bihemispheric processing. J Exp Psychol:HPP 18:763-784.

Barense MD, Bussey TJ, Lee AC, Rogers TT, Davies RR, Saksida LM, Murray EA, Graham KS. 2005. Functional specialization in the human medial temporal lobe. J Neurosci 25:10239-10246.

Barense MD, Gaffan D, Graham KS. 2007. The human medial temporal lobe processes online representations of complex objects. Neuropsychologia 45:2963-2974.

Barense MD, Henson RN, Lee AC, Graham KS. 2010a. Medial temporal lobe activity during complex discrimination of faces, objects, and scenes: Effects of viewpoint. Hippocampus 20:389-401.

Barense MD, Rogers TT, Bussey TJ, Saksida LM, Graham KS. 2010 b. Influence of conceptual knowledge on visual object discrimination: Insights from semantic dementia and MTL amnesia. Cereb Cortex 20:2568-2582.

Barense MD, Ngo JKW, Hung LHT, Peterson MA. Interactions of memory and perception in amnesia: the figure-ground perspective. Cereb Cortex (22:11), doi: 10.1093/cercor/bhr347.

Barense MD, Henson RN, Graham KS. 2011. Perception and conception: Temporal lobe activity during complex discriminations of familiar and novel faces and objects. J Cogn Neurosci 23:3052-3067.
Barense MD, Groen I, Lee AC, Yeung L, Brady S, Gregori M, Kapur N, Bussey TJ, Saksida LM, Henson RN. 2012. Intact memory for irrelevant information impairs perception in amnesia. Neuron 75:157-167.

Bartko SJ, Winters BD, Cowell RA, Saksida LM, Bussey TJ. 2007. Perirhinal cortex resolves feature ambiguity in configural object recognition and perceptual oddity tasks. Learn Mem 14:821-832.

Baxter MG. 2009. Involvement of medial temporal lobe structures in memory and perception. Neuron 61:667-677.

Bles M, Schwarzbach J, De Weerd P, Goebel R, Jansma B. 2006. Receptive field size-dependent attention effects in simultaneously presented stimulus displays. Neuroimage 30:506-511.

Bozeat S, Lambon Ralph MA, Patterson K, Garrard P, Hodges JR. 2000. Non-verbal semantic impairment in semantic dementia. Neuropsychologia 38:1207-1215.

Bright P, Moss HE, Stamatakis EA, Tyler LK. 2005. The anatomy of object processing: The role of anteromedial temporal cortex. Q J Exp Psychol B 58:361-377.

Bullier J. 2001. Integrated model of visual processing. Brain Res Rev 36:96-107.

Burke SN, Wallace JL, Nematollahi S, Uprety AR, Barnes CA. 2010. Pattern separation deficits may contribute to age-associated recognition impairments. Behav Neurosci 124:559-573.

Burke SN, Wallace JL, Hartzell AL, Nematollahi S, Plange K, Barnes CA. 2011. Age-associated deficits in pattern separation functions of the perirhinal cortex: A cross-species consensus. Behav Neurosci 125:836-847.

Bussey TJ, Saksida LM. 2002. The organization of visual object representations: A connectionist model of effects of lesions in perirhinal cortex. Eur J Neurosci 15:355-364.

Bussey TJ, Saksida LM, Murray EA. 2002. Perirhinal cortex resolves feature ambiguity in complex visual discriminations. Eur J Neurosci $15: 365-374$.

Clark RE, Reinagel P, Broadbent NJ, Flister ED, Squire LR. 2011. Intact performance on feature-ambiguous discriminations in rats with lesions of the perirhinal cortex. Neuron 70:132-140.

Cowell RA. 2012. Computational models of perirhinal cortical function. Hippocampus 22:1952-1964.

Cowell RA, Bussey TJ, Saksida LM. 2006. Why does brain damage impair memory? A connectionist model of object recognition memory in perirhinal cortex. J Neurosci 26:12186-12197.

Cowell RA, Bussey TJ, Saksida LM. 2010a. Components of recognition memory: Dissociable cognitive processes or just differences in representational complexity? Hippocampus 20:1245-1262.

Cowell RA, Bussey TJ, Saksida LM. 2010b. Functional dissociations within the ventral object processing pathway: Cognitive modules or a hierarchical continuum? J Cogn Neurosci 22:2460-2479.

Dale AM, Fischl B, Sereno MI. 1999. Cortical surface-based analysis. I. Segmentation and surface reconstruction. Neuroimage 9:179-194.

Driver J, Baylis GC. 1996. Edge-assignment and figure-ground segmentation in short-term visual matching. Cogn Psychol 31:248-306.

Fischl B, Sereno MI, Dale AM. 1999. Cortical surface-based analysis. II. Inflation, flattening, and a surface-based coordinate system. Neuroimage 9:195-207.

Fischl B, Liu A, Dale AM. 2001. Automated manifold surgery: Constructing geometrically accurate and topologically correct models of the human cerebral cortex. IEEE Trans Med Imaging 20:70-80.

Gibson BS, Peterson MA. 1994. Does orientation-independent object recognition precede orientation-dependent recognition? Evidence from a cueing paradigm. J Exp Psychol:HPP 20:299-316.

Graham KS, Barense MD, Lee AC. 2010. Going beyond LTM in the MTL: A synthesis of neuropsychological and neuroimaging findings on the role of the medial temporal lobe in memory and perception. Neuropsychologia 48:831-853.

Hellige JB, Taylor AK, Eng TL. 1989. Interhemispheric interaction when both hemispheres have access to the same stimulus information. J Exp Psychol:HPP 15:711-722. 
Henson RNA, Cansino S, Herron JE, Robb WG, Rugg MD. 2003. A familiarity signal in human anterior medial temporal cortex? Hippocampus 13:301-304.

Higuchi S, Miyashita Y. 1996. Formation of mnemonic neuronal responses to visual paired associates in inferotemporal cortex is impaired by perirhinal and entorhinal lesions. Proc Natl Acad Sci USA 93:739-743.

Insausti R, Juottonen K, Soininen H, Insausti AM, Partanen K, Vainio P, Laakso MP, Pitkanen A. 1998. MR volumentric analysis of the human entorhinal, perirhinal, and temporopolar cortices. Am J Neuroradiol 19:659-671.

Jenkinson M, Bannister PR, Brady JM, Smith SM. 2002. Improved optimization for the robust and accurate linear registration and motion correction of brain images. Neuroimage 17:825841.

Kastner S, De Weerd P, Desimone R, Ungerleider LG. 1998. Mechanisms of directed attention in the human extrastriate cortex as revealed by functional MRI. Science 282:108-111.

Kastner S, de Weerd P, Pinsk MA, Elizondo MI, Desimone R, Ungerleider LG. 2001. Modulation of sensory suppression: implications for receptive field sizes in the human visual cortex. J Neurophysiol 86:1398-1411.

Kim S, Jeneson A, van der Horst AS, Frascino JC, Hopkins RO, Squire LR. 2011. Memory, visual discrimination performance, and the human hippocampus. J Neurosci 31:2624-2629.

Kirchner H, Thorpe SJ. 2006. Ultra-rapid object detection with saccadic eye movements: Visual processing speed revisited. Vis Res 46:1762-1776.

Kohler S, Danckert S, Gati JS, Menon RS. 2005. Novelty responses to relational and non-relational information in the hippocampus and the parahippocampal region: A comparison based on eventrelated fMRI. Hippocampus 15:763-774.

Lambon Ralph MA, Patterson K. 2008. Generalization and differentiation in semantic memory: Insights from semantic dementia. Ann NY Acad Sci 1124:61-76.

Lamme VAF, Roelfsema PR. 2000. The distinct modes of vision offered by feedforward and recurrent processing. Trends Neurosci 23:571-579.

Lancaster JL, Woldorff MG, Parsons LM, Liotti M, Freitas CS, Rainey L, Kochunov PV, Nickerson D, Mikiten SA, Fox PT. 2000. Automated Talairach atlas labels for functional brain mapping. Hum Brain Mapp 10:120-131.

Lee AC, Bussey TJ, Murray EA, Saksida LM, Epstein RA, Kapur N, Hodges JR, Graham KS. 2005. Perceptual deficits in amnesia: Challenging the medial temporal lobe 'mnemonic' view. Neuropsychologia 43:1-11.

Lee AC, Rudebeck SR. 2010. Human medial temporal lobe damage can disrupt the perception of single objects. J Neurosci 30:65886594.

Lee AC, Yeung LK, Barense MD. 2012. The hippocampus and visual perception. Front Hum Neurosci 6:91.

Levy J, Trevarthen C. 1976. Metacontrol of hemispheric function in human split-brain patients. J Exp Psychol:HPP 2:299-312.

Liu Z, Richmond BJ. 2000. Response differences in monkey TE and perirhinal cortex: Stimulus association related to reward schedules. J Neurophysiol 83:1677-1692.

Marsolek CJ. 1999. Dissociable neural subsystems underlie abstract and specific object recognition. Psychol Sci 10:111-118.

McTighe SM, Cowell RA, Winters BD, Bussey TJ, Saksida LM. 2010. Paradoxical false memory for objects after brain damage. Science 330:1408-1410.

Murray EA, Bussey TJ, Saksida LM. 2007. Visual perception and memory: A new view of medial temporal lobe function in primates and rodents. Annu Rev Neurosci 30:99-122.

Murray EA, Wise SP. 2012. Why is there a special issue on perirhinal cortex in a journal called Hippocampus? The perirhinal cortex in historical perspective. Hippocampus 22:1941-1951.
Naya Y, Yoshida M, Miyashita Y. 2001. Backward spreading of memory-retrieval signal in the primate temporal cortex. Science 291:661-664.

Naya Y, Yoshida M, Miyashita Y. 2003. Forward processing of longterm associative memory in monkey inferotemporal cortex. J Neurosci 23:2861-2871.

Newsome RN, Duarte A, Barense MD. 2012. Reducing perceptual interference improves visual discrimination in mild cognitive impairment: Implications for a model of perirhinal cortex function. Hippocampus 22:1990-1999.

Patterson K, Lambon Ralph MA, Jefferies E, Woollams A, Jones R, Hodges JR, Rogers TT. 2006. "Presemantic" cognition in semantic dementia: Six deficits in search of an explanation. J Cogn Neurosci 18:169-183.

Peterson MA, Gibson BS. 1994a. Must figure-ground organization precede object recognition? An assumption in peril. Psychol Sci 5:253-259.

Peterson MA, Gibson BS. 1994b. Object recognition contributions to figure-ground organization: Operations on outlines and subjective contours. Percept Psychophys 56:551-564.

Peterson MA, Gerhardstein PC, Mennemeier M, Rapcsak SZ. 1998. Object-centered attentional biases and object recognition contributions to scene segmentation in left- and right-hemisphere-damaged patients. Psychobiology 26:557-570.

Peterson MA, de Gelder B, Rapcsak SZ, Gerhardstein PC, BachoudLévi A-C. 2000. Object memory effects on figure assignment: Conscious object recognition is not necessary or sufficient. Vis Res 40:1549-1567.

Peterson MA, Harvey EH, Weidenbacher HL. 1991. Shape recognition inputs to figure-ground organization: Which route counts? J Exp Psychol 17:1075-1089.

Peterson MA, Enns JT. 2005. The edge complex: Implicit memory for figure assignment in shape perception. Percept Psychophys 67:727740 .

Peterson MA, Skow E. 2008. Inhibitory competition between shapes properties in figure-ground perception. J Exp Psychol:HPP 34:251-267.

Rogers TT, Lambon Ralph MA, Garrard P, Bozeat S, McClelland JL, Hodges JR, Patterson K. 2004. Structure and deterioration of semantic memory: A neuropsychological and computational investigation. Psychol Rev 111:205-235.

Romberg C, McTighe SM, Heath C, Whitcomb D, Cho K, Bussey TJ, Saksida LM. 2012. False recognition in a mouse model of Alzheimer's disease: Rescue with sensory restriction and memantine. Brain 135:2103-2114.

Serre T, Oliva A, Poggio TA. 2007. A feedforward architecture accounts for rapid categorization. Proc Natl Acad Sci 104:6424-6429.

Scalf PE, Banich MT, Kramer AF, Narechania K, Simon CD. 2007. Double take: Parallel processing by the cerebral hemispheres reduced the attentional blink. J Exp Psychol:HPP 33:298-329.

Scalf PE, Banich MT, Erickson AB. 2009. Interhemispheric interaction functionally expands attentional capacity: Evidence from a selective attention task in the auditory modality. Exp Brain Res 194:317-322.

Scalf PE, Beck DM. 2010. Competition in visual cortex impedes attention to multiple items. J Neurosci 30:161-169.

Scalf PE, Beck DM. 2011. The effects of dividing attention on target enhancement and distractor suppression. J Vis 11:104.

Sereno MI, Dale AM, Reppas JB, Kwong KK, Belliveau JW, Brady TJ, Rosen BR, Tootell RBH. 1995. Borders of multiple visual areas in humans revealed by functional magnetic resonance imaging. Science 268:889-893.

Smith SM, Jenkinson M, Woolrich MW, Beckmann CF, Behrens TEJ, Johansen-Berg H, Bannister PR, De Luca M, Drobnjak I, Flitney DE, Niazy R, Saunders J, Vickers J, Zhang Y, De Stefano N, Brady JM, Matthews PM. 2004. Advances in functional and structural MR image analysis and implementation as FSL. Neuroimage 23:208-219. 
Squire LR, Zola-Morgan S. 1991. The medial temporal lobe memory system. Science 253:1380-1386.

Squire LR, Wixted JT. 2011. The cognitive neuroscience of human memory since H.M. Annu Rev Neurosci 34:259-288.

Straw AD. 2008. Vision egg: An open-source library for realtime visual stimulus generation. Front Neuroinform 2:4, 1-10.

Suzuki WA. 2009. Perception and the medial temporal lobe: Evaluating the current evidence. Neuron 61:657-666.

Suzuki WA, Amaral DG. 1994. Perirhinal and parahippocampal cortices of the macaque monkey: Cortical afferents. J Comp Neuro 1350:497-533.
Takeuchi D, Hirabayashi T, Tamura K, Miyashita Y. 2011. Reversal of interlaminar signal between sensory and memory processing in monkey temporal cortex. Science 331:14431447.

Woolrich MW, Ripley BD, Brady JM, Smith SM. 2001. Temporal autocorrelation in univariate linear modeling of FMRI data. Neuroimage 14:1370-1386.

Xiang JZ, Brown MW. 1998. Differential neuronal encoding of novelty, familiarity and recency in regions of the anterior temporal lobe. Neuropharmacology 37:657-676. 